\title{
Say It with an App
}

\author{
Cleto Corposanto ${ }^{1^{*}}$ and Beba Molinari ${ }^{2}$ \\ ${ }^{1}$ Department of Legal, Historical, Economic and Social Science, Catanzaro , Italy \\ ${ }^{2}$ Department of Educational Science, Genova, Italy \\ Email: cleto.corposanto@unicz.it, beba.molinari@unige.it
}

\begin{abstract}
This paper aims to explore the new opportunities offered by the web 2.0, with particular emphasis on the level of intrusiveness concerning web surveys and their different methods of administration. Particular attention will be drawn to the Apps and the way they intervene in the research design to give the researchers the opportunity to learn about the preferences and the consultations which are mostly relevant to the users, thanks to the analysis carried out by data mining and web survey.
\end{abstract}

Keywords: App, web survey, big data, web research, new media, data mining.

\section{Introduction}

Every day we deal more and more with a large amount of information gathered at anytime from anywhere via Smartphone, tablets and notebooks. Indeed, today the web is not only a virtual place to access from a fixed location, but it also represents an opportunity for people willing to exchange information. It works as a showcase or even a community where everyone can share opinions and files of any kind. On account of this, we sought to identify the new opportunities offered by the web - seen as a generator of big data - as well as the new techniques provided by the web research[1]. We gave particular emphasis to the "APPlications" (Apps), which can be easily installed on any device be it a Smartphone or a tablet ${ }^{1}$. These are generally considered as a means aimed at satisfying an immediate need, such as checking the weather forecasts, accessing to social media, playing videogames. However, we often forget about the versatility of the web[2], which can also serve as a tool for collecting data. That is to say, it can be concluded that the web is not only an essential means of retrieving texts and articles. It is rather a modern tool used to gather data through online platforms.

Over the last years, several studies, from marketing research[3] to thesis and dissertations, have been conducted using web surveys. This has led us to investigate on the different levels of intrusiveness of these new technologies, by taking into consideration both the traditional web surveys as well as the "Apps", the latter being more commonly used in everyday life than in the research. Thanks to these new technologies, researchers have had the opportunity to learn about the users' preferences and consultations. In addition, they have obtained personal and relational data which would be normally retrieved through the administration of traditional paper and pen surveys[4], or web surveys[5].

All things considered, a first investigation was carried out by the Observatory on the quality of celiac patients' life[6]. Thanks to the analysis of data gathered from an App, it has been possible to learn about the preferences of celiac patients following a gluten-free diet, which is, nowadays, one of the new treatments to prevent such a disease. In addition, a web survey regarding the dietary compliance of people affected by CD was further discussed[7][8][9], in order to compare the level of intrusiveness between the two studies. The outcomes obtained from the analysis have been interpreted with the aim to shed light on the quality of the data, which is partly determined by the different levels of intrusiveness emerging from the use of these two distinct data collection tools. These can be considered

1 The definition adopted by the Food and Drug Administration (2014) concerning mobile apps is the following: "Mobile apps are software programs that run on smartphones and other mobile communication devices. They can also be accessories that attach to a smartphone or other mobile communication devices, or a combination of accessories and software. 
complementary and they both enable us to better understand the wide scenario concerning the celiac disease.

In the light of the above considerations, the aim of this contribution is to understand whether the administration of online questionnaires by means of different devices such as online platforms and Apps can be key factors when it comes to issues concerning the concept of intrusiveness, and if such devices are considered to be different in their function.

Finally, particular attention has been given to the concept of 'quantitative research'. Specifically, it is important to understand whether the researchers should maintain an approach based on the relational production of the basic data for the formulation of their assumptions[10], or they need to reconsider this aspect by virtue of the new research tools $[9]$.

\section{A Few Considerations on the Method}

Great attention has been devoted to the level of intrusiveness within the traditional contexts of research [11]. However, there has been too little discussion of the different methods, techniques and objects of study concerning web 2.0.

In the first place, the concept of intrusiveness refers to the extent to which the researcher comes into contact with the object of study and how this latter can be somehow influenced. So, a first aspect to be considered is to verify whether the contact with the researcher may alter the results and if there are objects of study and/or particular settings that better adapt to intrusions, without forgetting that the effects of disturbance are always present and can incur consistently according to the tool used to collect data. This helps us become aware of the existence of both intrusive and non-intrusive techniques ${ }^{2}$. This latter aspect, will be further discussed in the following pages be it considered either as a dichotomy, with presence/absence of disturbance effects[11][12], or a space-time continuum, where the 'nonintrusiveness' is designed as a second-level concept[13][14].

The above mentioned aspects bring us back to the longstanding question of privacy. So far people have been required a written consent before completing a survey. However, the current situation regarding the e-research seems to be more complicated. On account of this we should ask ourselves whether the rules adopted in traditional contexts are still valid, or if they need to be revised according to the website policies. This consideration is only one of the many questions arisen in the course of our study. Another aspect to take into account concerns the rules that define the procedures in the field of research according to the areas covered and developed in the study: suffice it to think of international studies with specific legislative peculiarities, maybe even within different areas of specialization (medicine, sociology, psychology). What approach and what standards on privacy should be adopted in this case? The so called Ethics Committees[15] were set up to respond properly to these issues and examine all the possibilities emerged from the various research contexts. In our specific case study regarding Apps and web-surveys it was not necessary to involve such an entity, for some reasons which will be further discussed. On the contrary, in a purely medical study, where patients' medical records needed to be analysed, each medical device requested the approval from its own ethics committee membership to allow the research to be conducted. Before that, the research organization had to define a priori its own code of ethics, the informed consent as well as the confidentiality of data[16]. With respect to the good practices to be adopted, the Oxford University was the first to sign, jointly with the University of Amsterdam, an official document providing the proper guidelines to be followed in the emethods ${ }^{3}$ - based studies.

In the next section, we will focus on the choices taken in the course of our study as regards the issues concerning the confidentiality of data, the anonymity, the consumer profiling of the users along with the respective informed consent[17].

${ }^{2}$ In the early sixties the concept of intrusiveness already played an important role. A clear example is a text of Eugene Webb, Donald Campbell, Richard Schwartz e Lee Sechrest "Unobtrusive Measures: Non-Reactive Research in the Social Sciences" (1966), in which the importance of the non-intrusive techniques within the research design was discussed.

3 The document can be downloaded from the following link: http://eresearch-ethics.org 
Given such methodological premises, before discussing the final achievements, it is important to make a better understanding of the reasons that led us to consider the possibility of comparing these studies which are both related to the world of celiac disease.

The first case considered aims at investigating the phenomenon of dietary compliance by deliberate choice, in order to define the eating habits and the dietary transgression that portray the quality of life of celiac patients. Almost 6,000 people participated in the survey and the administration of the questionnaire was primarily divulged from the AIC amongst its associated parties and on Twitter and Facebook pages, on the personal profiles of the association and the researchers.

As regards the use of Apps, it can be argued how data collected for secondary purposes bring about a transversal interpretation to define the preferences and analyze the lifestyles of people affected by celiac diseases. Indeed, what is important to emphasize is not so much the nature of the App's contents, which fosters a clear innovation as to the analysis of data, rather the opportunity to access a range of information which otherwise would not be available except through an ad hoc study[9].

In particular we analyzed the results obtained from an investigation issued by the big data collected through an App that supports people with celiac disease when eating out or purchasing adequate food at the supermarket for a gluten free diet. Simultaneously, thanks to this mobile device, a mini web survey was administered.

That is to say, it can be assumed that both studies bring about a twofold objective. On one hand, great attention will be given to the functionality provided by the administration of web surveys through the online platforms. In the second case, we will observe the secondary data obtained on the basis of the parameters defined by the App as well as the aspects related to the accesses and the compilations of a web survey administered through this mobile device.

From a purely theoretical point of view, as it is shown in the diagram below, we can distinguish two macro areas with respect to the use of mobile devices: if on the one hand we can avail ourselves as simple end-users of the App ${ }^{4}$, on the other hand we may use them as a tool for "ad hoc" surveys 5 .

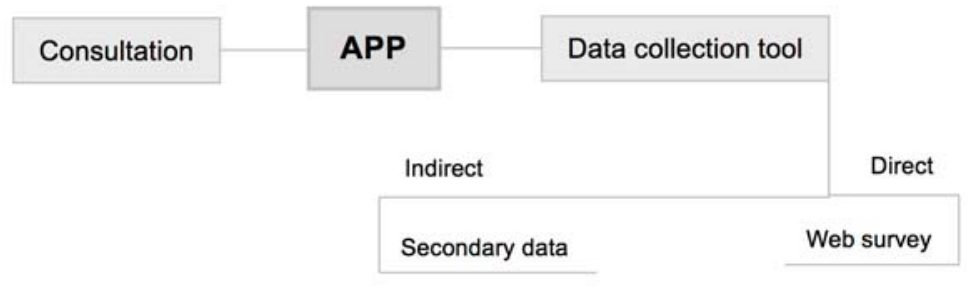

Figure 1. Mobile devices as a tool for collecting data

In this second case researchers would have access to a number of big data which can be made available in a direct or indirect manner. Indirect, since the platform is structured in such a way as to collect by itself the data: the researcher is provided with a database, where he can retrieve all the data concerning the geographical area, the services and/or products consulted, the IP addresses accessing the survey as well as the device set up to connect to the App, be it a Smartphone or a tablet.

Finally, we find it worth noting that the two studies in comparison are both linked to the concept of m-health. The term refers to all those mobile devices which support the practice of medicine, the health and the well-being of patients[19]. Naturally the main difference is determined by the form of the online questionnaire but the main focus will be now shifted to the big data and the way a means such as a web survey may become supported for a mobile device.

${ }^{4}$ In addition to the most commonly used devices such as Smartphone and tablets, the App can be found on PDAs, laptops, mp3 players, GPS receivers and on all mobile computing devices.

${ }^{5}$ Before discussing this last aspect it is important to highlight the presence of a sine qua non condition stating that only the "owner" i.e. the designer and executer is responsible for the inclusion of the survey into the App 


\section{$3 \quad$ Web Survey and App: Technical Intrusive or Not Intrusive?}

In the present paper, we will take into consideration the decisions made regarding the confidentiality of the data, namely how much and by virtue of what the intrusiveness level varies according to the device in use $^{6}$.

From the study on the dietary compliance ${ }^{7}$ it has become clear that over the last years, people with celiac disease have increased their level of awareness and knowledge about the risks arising after the digestion of gluten. As a matter of fact, the higher the level of awareness about one's eating habits, the lower the possibility to adopt a risky behavior.

The two surveys carried out in 2012 and 2013 show that the results are not significantly different; $89 \%$ of the participants do not consume food out of their gluten free diet, with a favorable deviation of $3 \%$ between the second and the first survey. From the observation of the findings concerning the eating behavior and the temptation to transgression, it is worth noting a grey area, which was also mentioned in the previous survey.

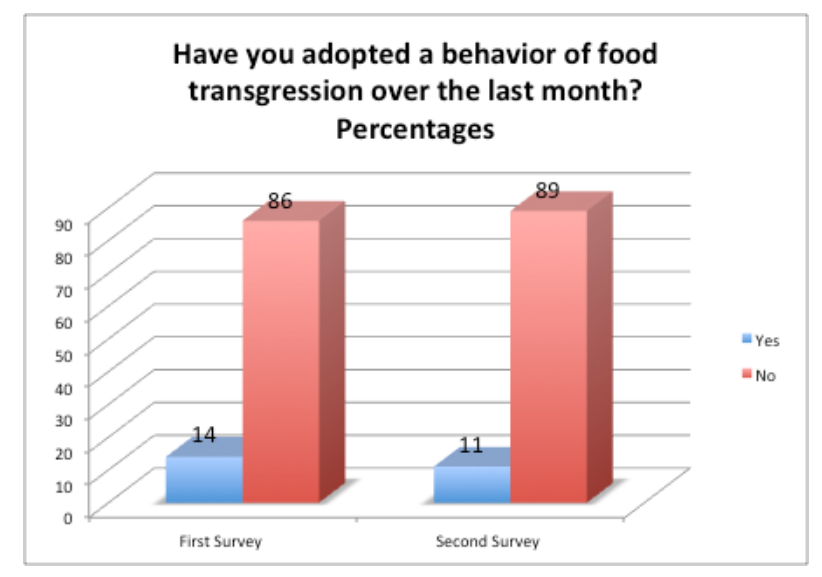

Figure 1. Dietary transgression over the last month - Survey 2011-2013 - Percentages

Given that $14 \%$ of patients have declared to have transgressed at least once over the last month and $47 \%$ feel the need to do it every now and then, we can therefore postulate the existence of a $33 \%$ grey area, which induces us to ascertain the reasons that have led these people to avoid a dietary transgression.

In this regard, it is important to stress out that the grey area has decreased from $37 \%$ to $33 \%$ with $4 \%$ fluctuation. This confirms that people affected by CD have arisen awareness as to their living conditions. Moreover, we must point out that losing 4 percentage points in two years is quite a good result, if we think that not only habits but also social conventions, which are usually difficult to abandon, have been somehow touched.

Although the study group seems to be particularly aware of the risks posed by gluten ingestion than in the past, only 5\% decide to consult the doctor. Family involvement settles at $23 \%$ whereas the number of people who decide not to inform anybody increases by $5 \%$ improving from $18 \%$ to $23 \%$.

Getting back to the lifestyles adopted by people with celiac disease, it turns out that $19 \%$ of those who transgress feel guilty, confirming their awareness of having committed an action that can affect their health. $10 \%$ are apparently not concerned and only $2 \%$ consider it as a pleasant action. In addition to this, the chart shows how, within two years, the approach to celiac disease has changed. In fact, on one hand, nearly half of the participants consider transgression as unnecessary and insignificant; the other half, on the contrary, seems not to assess the risk incurred by breaking the diet.

${ }^{6}$ Regarding the study on the dietary compliance, it is advisable to read Corposanto e Molinari (2013b).

${ }^{7} 3,000$ people with celiac disease responded to the first version of the survey whereas 147 fewer people participated in the second one. 
As regard the level of intrusiveness, instead, it can be assumed that the web survey has brought about a lower intrusion by the researcher, if compared to a traditional survey. In fact, on one hand the questionnaire realized by the researcher has maintained a high level of intrusiveness because he decides previously the variables to include, on the other hand, the platform has not allowed to interact with the respondents, thus reducing to a minimum the level of social desirability. In any case, the participant is guided to complete the survey by using "pop ups", in order to face the difficulties arisen by the diverse response modalities.

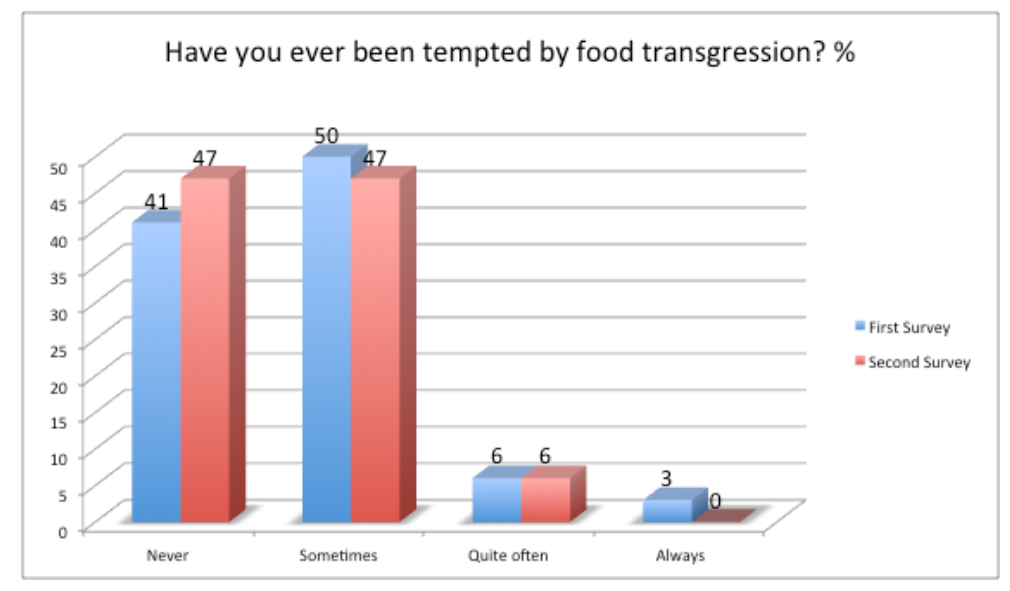

Figure 2. How many times a day a person affected by CD thinks about dietary transgression Survey 2011-2013 - Percentages

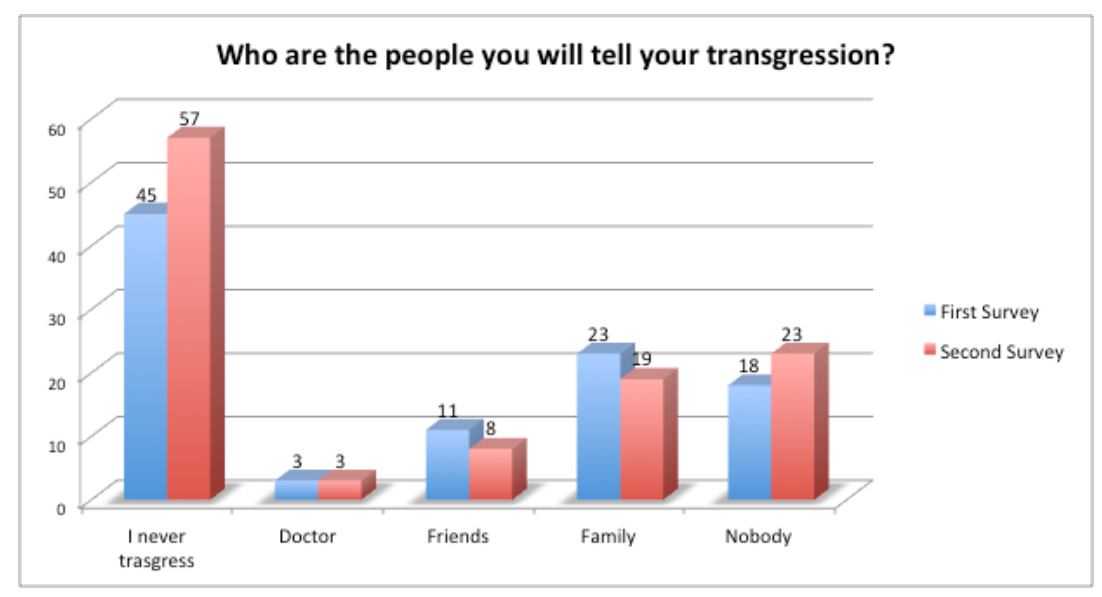

Figure 3. Who are the people you will tell your transgression? - Survey 2011-2013 - Percentages

With respect to the ethical nature of the research and the procedure provided by the web survey, we consider it essential, to make the users who accesses the questionnaire through a link aware of some steps to be followed. The first page, which includes the link that connects to the site, draws attention to the purpose of the study as well as the analysis and the publication of the findings which will be shown and discussed without any direct reference to the individual respondent. Before accessing the survey however, it is essential to read carefully the informed consent and click on the box below to consult the privacy policy. Once these steps have been completed, a new screen will open to start completing the survey.

In the latest generation of platforms it has been possible to make sure that the IP address from which the respondents $\log$ on are not identified. This aspect can be further stated within the informed consent, 
so that even the IT specialists may be able to acknowledge that anonymity is safeguarded. However, [17] claims that among the ethical aspects there might exist the traceability of the consumer's profile, i.e. the so-called web-surfers profiling. This condition, however, is not directly connected with this kind of studies as it does not fall within the objectives of the research. It might rather be an issue of more considerable interest with regard to the meta-data collected through the Apps.

Returning to our study at hand, in the first place we carried out a web survey on dietary compliance, administered through an online platform; afterwards we conducted a first preliminary analysis of the meta data collected by default by the application and another mini web survey aimed at investigating the level of satisfaction with respect to the mobile device.

If we drew an imaginary line we would think that the survey carried out by App is an advanced version of the previous case as it utilizes most of the modern concepts of interactivity among the new media. Actually, the real strength of both studies is represented by the use of a web platform. This enables us to analyze the collected data and provides us with real-time big data in a series of summary reports indicating vices and virtues ${ }^{8}$.

To get straight to the heart of our research carried out by means of the App, we find it important to draw attention to the large number of people involved in the project thanks to the use of the above mentioned tools. Just one month since the launch of the App, the first version for iPhone was downloaded by nearly 20,000 users, whereas the version for Android was installed on 14,000 mobile devices, where the end users amount to approximately 8,000. A real "data explosion"[19] is achieved, which is quite difficult through the most classical tools, unless we decide to conduct proper census during the phase of data collection considering both advantages and disadvantages that may incur (Molinari, 2013).

To discuss the meta data collected by default from the App, we can come to the conclusion that the total number of screenings per keyword done on the iPhone version reached about 28,000 researches in just over a month, which is equivalent to $78 \%$ of the total of what had been consulted on mobile devices. This is likely to confirm the trend of an increased use of mobile phones for specific areas of interest such as nutrition.

Table 1. From the App Google Analytics - survey on foods -

\begin{tabular}{lcccccc}
\hline \multicolumn{1}{c}{ Search term } & $\begin{array}{c}\text { Total } \\
\text { research }\end{array}$ & Page views & \% drop out & $\begin{array}{c}\text { Research } \\
\text { completion }\end{array}$ & $\begin{array}{c}\text { Time after } \\
\text { the } \\
\text { research }\end{array}$ & $\begin{array}{c}\text { Research } \\
\text { depth }\end{array}$ \\
& 31.722 & 1,09 & $34,30 \%$ & $47,20 \%$ & $00: 10: 03$ & 1,11 \\
& total $\%$ & Mo web & Averege & Averege & Averege & Averege \\
& 100,00 & site: 1,09 & $34,30 \%$ & $47,20 \%$ & $00: 10: 03$ & 1,11 \\
& $(31.722)$ & $(0,00 \%)$ & $(=0,00)$ & $(0,00)$ & $(0,00 \%)$ & $(0,00 \%)$ \\
Brand of Crisps & 328 & 1,05 & 64,02 & 29,15 & $00: 13: 30$ & 0,39 \\
Brand of ice-cream & 244 & 1,03 & 69,67 & 20,24 & $00: 09: 36$ & 0,73 \\
Brand of ice-cream & 233 & 1,06 & 62,23 & 28,34 & $00: 10: 47$ & 0,72 \\
Candies & 258 & 1,07 & 36,08 & 43,79 & $00: 04: 56$ & 1,35 \\
Cream & 163 & 1,09 & 33,33 & 45,51 & $00: 07: 49$ & 1,29 \\
Yeast & 136 & 1,12 & 47,06 & 40,79 & $00: 15: 55$ & 1,05 \\
Chips & 131 & 1,08 & 34,35 & 48,92 & $00: 20: 36$ & 1,20 \\
Mayonnaise & 121 & 1,05 & 43,80 & 40,16 & $00: 01: 58$ & 0,99 \\
Brand of ice-cream cake & 119 & 1,07 & 64,71 & 31,50 & $00: 16: 12$ & 0,32 \\
Brand of fruit juice & 119 & 1,11 & 62,18 & 33,33 & $00: 10: 57$ & 0,47 \\
\hline
\end{tabular}

${ }^{8}$ For an in-depth study, [9], where strengths and weaknesses regarding online statistical analysis have been sufficiently detailed 
Table 1 highlights the nutritional aspects and provides a range of information as regards the kind of diet followed by people affected by $\mathrm{CD}^{9}$. It focuses, for instance, on the brands, the market and the macro-categories of products which are particularly attractive for this segment of population. In fact, the use of the App has sharply increased over the last three years amongst celiac patients and their family members. The analysis of the big data concerning the foods (Table 1.), confirms what emerged in the above mentioned web survey on dietary compliance[8], with the addition of particular details about eating habits. Great attention has been devolved to the product-by-product search for food, stressing the difficulties of abandoning the unhealthy eating habits regarding the consumption of 'junk food' such as chips, crisps, mayonnaise, ice creams. Obviously, this kind of information may become useful if provided in support of an ad hoc study, as in the case of the web survey on dietary compliance, where specific questions about foods and preferences were posed ${ }^{10}$.

This type of data is not required by the researcher as they are collected directly from the App. That is to say that the level of intrusiveness is almost null.

We now try to understand how much the use of the App, to investigate on the meals consumed out of home, has been appreciated by people with celiac disease. In this respect we carried out a web survey to inspect if and to what extent such new tools are considered useful. It emerges that $3 / 4$ of the sample has been consulting the list for at least two years, and in $38 \%$ of the cases at a frequency of at least once per month $^{11}$.

If in addition we were to investigate on patients' favorite consultation tools such as printable or online guides made also available onto the mobile devices, we would note a substantial increase from $0 \%$ to $24 \%$ in the use of the App, which since the moment they were launched in Summer 2011, have probably been the tool employed by almost a quarter of the respondents. The modality of consulting by means of IT tools, such as personal computers and mobile applications encountered considerable interest from almost half of the study participants (46\%). Banking on these results, we can confirm that such data are fully in line with the statement about the growing use of new technologies in the daily life by the majority of the world's population[20].

\section{Consultation/navigation of the guide}

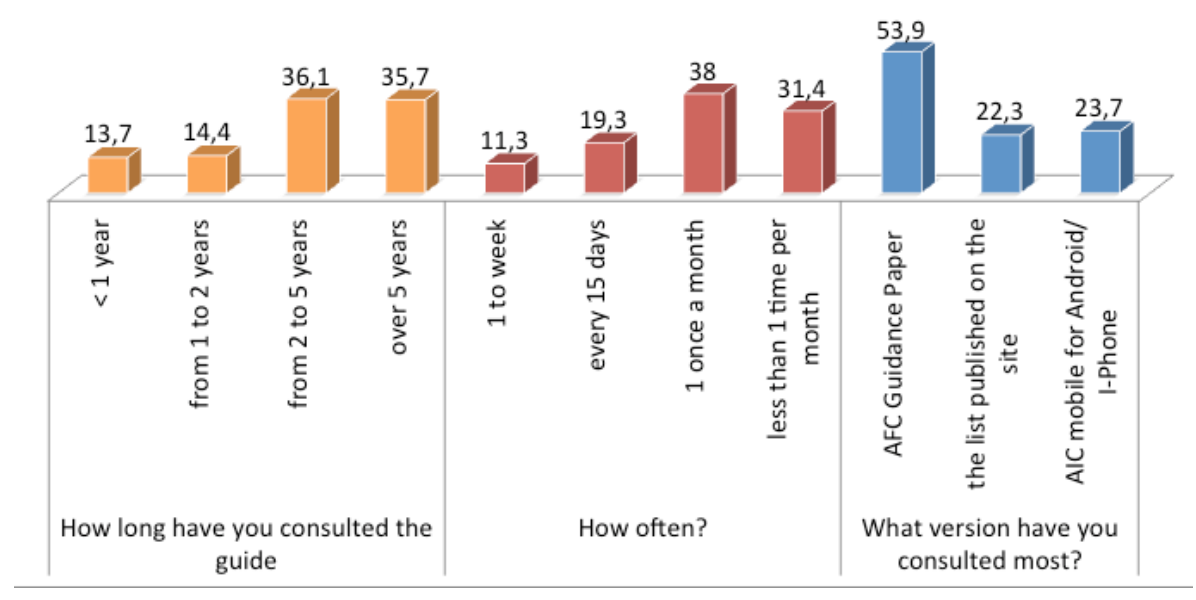

Figure 4. Preferences expressed through web survey with reference to the App- Percentages

To estimate the level of intrusiveness with respect to the web-survey concerning the App users satisfaction, we adopted the same procedure described above for the study on the dietary compliance. As

${ }^{9}$ As to the products considered by the users, all the brands related to the names of supermarket chains were modified to be displayed with the "specific brand" notice.

${ }^{10}$ For more information concerning food preferences in relation to meals namely breakfast, lunch, afternoon break, dinner, please consult the following contribution, [7].

11 1,430 App users participated in the survey 
regards the mega data shown in Figure 2 and 3 we can assume that the situation has slightly changed but it should not be reason of concern, if we consider the user as the App's owner. Once the service has been provided, people affected by CD will be able to download the App from the web, after which an informed consent along with the privacy police acknowledgement will be requested. Once these passages have been fulfilled, the user will be able to use the App functions. However, when new functions are introduced, including those typically related to research contexts such as a web survey administered by the App itself, a privacy consent will be further required. At this point, it will be essential to specify the purpose of the survey, the name of the person in charge of collecting data and their use.

\section{Final Considerations: Non-Intrusiveness in the Web 2.0}

With respect to our initial assumption, according to which the studies presented intended to investigate whether the administration of online questionnaires through different devices, such as platforms for web survey and App, may intervene in the concept of intrusiveness and if such devices can be considered as two distinct detection tools, we can outline some considerations in the light of what has been revealed above.

Before coming to a conclusion, however, it is useful to distinguish some aspects concerning the concept of intrusiveness. On one hand this paper has drawn particular attention among the different devices used to administer online questionnaires, being web survey to be completed at fixed locations or through mobile devices. On the other hand, the big data available through web analytics have been also shown.

With reference to the first study, where the concept of intrusiveness is considered as a dichotomy i.e. presence-absence of the variable[11][12] or as a space-time continuum [14], several aspects regarding the two devices taken into consideration can be further discussed. At first we attempted to prove whether some tools are more intrusive than others. In reality, it is important to make clear that the researcher shows a direct interest in the interviewees. Therefore it is quite normal for the respondents to provide answers to the specific questions posed, which are mediated by the phenomenon of "social desirability".

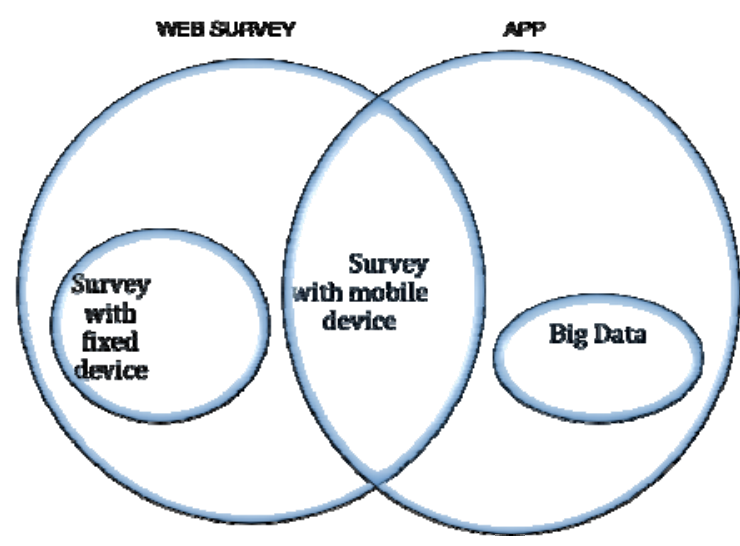

Figure 5. Difference between Web Survey \& App

At the same time, as a result of the use of devices, there seems to be a lack of direct contact between interviewers and interviewees who are, in both studies, separated by a monitor. The assumption that a screen may play a mediator role between the two parties and therefore reduce possible elements of intrusiveness, such as the non verbal language, which could easily escape unexperienced or inattentive interviewers, leads us to make a further remark. This concerns, in particular, the setting where the compilation of the survey takes place, which is important and very different in the case of web surveys and questionnaires completed by means of an App. As a matter of fact both compilations take place online but in different contexts. Indeed, web surveys are likely to be more accurate as it is the respondent who decides, from a fixed location, the way and time to access. Probably, this leads him/her 
to focus better on the completion of the questionnaire. On the contrary, as regards surveys completed through the App, respondents can only postpone the moment. In addition, given the size of the screen where the questionnaire is displayed as well as the lack of a fixed location, we dare to conclude that the level of attention as to the completion of the survey through App is significantly lower than in the case of web survey. A further element of differentiation compared to traditional data collection means is the language used in the formulation of questions. Considering the methodological rules concerning the questionnaires, it can be observed that the language used when the user accesses the web is extremely direct and immediate; this aspect is mainly concerned with the web survey. On the contrary, as regards the surveys administered through App, the questions should be read in its entirety, by limiting, as far as possible, the number of characters because of the lack of space related to the screen size and resolution.

All things considered, we can consider overcome aspects that consider the intrusiveness as a dichotomous variable [11] and the space-time continuum approach[14] is, in our opinion a difficult tightrope even in the present case. However, what we want to bring to light is that through the web it is possible to access a larger number of compilations than through paper surveys. Furthermore, we must not forget the Central Limit Theorem stating that given a sufficiently large sample size from a population with a finite level of variance, the mean of all samples from the same population will be approximately equal to the mean of the population (with mean $\mu$ and variance $s / \sqrt{n}$ ), where $n$ is the number of samples. At another time and place ${ }^{12}$ we have proved that, after having calculated the sampling error and considered other aspects such as the phenomena of break off and drop out, the study on the dietary compliance presents a sampling error amounting to $1,74 \%$ with a confidence interval of $(1,96)$.

Last but not least, the next issue to be considered concerns some observations regarding the big data, which is the use of secondary data which in our study have been retrieved from mobile applications. Undoubtedly, we have been given the opportunity to become familiar with brands and gluten free products and identify restaurant owners providing gluten free foods. Thanks to these data it has been possible to share a different view that we would not have noted if we had used the traditional collecting techniques. This was only possible thanks to the "data mining", i.e. the extraction and the consequent analysis of data in the database which allowed the management of a statistical and mathematical amount of information that would otherwise be stored and considered as "statistical noise"[21].

With reference to the a greater or lesser intrusiveness of the big data generated during the app, we can say that these data are not intrusive, because derived spontaneously from a direct request of the person using the app. the researcher intervenes at a later stage, during the phase of the data analysis. We are discussing the so-called "web analytics" [22](Kaushik, 2010), that the analyzes carried out on individual queries that users perform during use the app, and where, thanks to login, you can have more information also kind of an identity In Italy, the theory of "confidentiality doctrine" has not been discussed yet. On the contrary, this concept based on the control over the data gathered indirectly from the web has become very popular in the USA[23].

We turn it upside down so the discussion and think about the intrusiveness watching from the analysis and the next step of publicizing the results Think of a box of tools web 2.0 means to define the frontiers of research. you need to discover new areas of study, considering the existing data banks with which we are not used to confront ourselves. This data when analyzed with the appropriate instruments become real matrices on which carrying out the analysis we consider most appropriate.

So in conclusion do quantitative research today, from our point of view, means to enrich their tool box and be able to draw on other Web 2.0 tools, including precisely the web-survey and app, the approach to co-creation information elementary [10] does not change, the quality of the data is valid, it will change the shape with which you study the data, the tools are different as well as the analyzes ${ }^{13}$.

12 Conference entitled "Issues concerning sampling and collection in online surveys and extensive traditional research" dated 26 September 2014 at the Catholic University of Milan.

13 Make reference to the publication of the proceedings of the conference "The challenge of the Italian health system: between structural crisis and social innovation" Rome, 29th may 2014, where the following contribution was discussed: "the toolbox 2.0". 
Naturally, the use of an App for research purposes is not only limited to people affected by celiac diseases. The object of study is very extensive, and the healthcare sector is just one example to be considered for this kind of research. Plenty of Apps have become a great support for medical devices.

The use of applications within the medical field has been expanding, to the extent that in 2013, the Food and Drug Administration drafted a document containing a set of guidelines[18].

In conclusion, thanks to Web 2.0, we have the great opportunity to make use of different software such as online platforms for web survey, App, social media, database, ad hoc programs. Such new technologies enable people to study the network by going hand in hand with the society.

\section{References}

1. J. Bethlehem and S. Biffignandi, "Handbook of Web Surveys. Wiley Handbooks in Survey Methodology", John Wiley \& Sons, 2012.

2. C. Cipolla, "Perché non possiamo non essere eclettici", Franco Angeli, 2013.

3. A. Arvidsson and A. Delfanti, "Introduzione ai media digitali", Il Mulino, 2013.

4. R. Bichi, "La conduzione delle interviste nella ricerca sociale", Roma 2007.

5. D.A. Dillman, "Mail and Internet Surveys: The Tailored Design Method" (2nd Edition ed.), John Wiley \& Sons, 2006.

6. S. Neuhold and B. Molinari, "Guida AFC, per metà consultata online o con App. I risultati del sondaggio su gradimento e fruibilità", in Celiachia Notizie, Anno XXXII N.83, 2013.

7. C. Corposanto and B. Molinari, "Quando il dover mangiare in modo diverso complica le relazioni sociali", in c. Clemente, P.P. Guzzo, "I sistemi sociosanitari regionali tra innovazioni e spendibilità. Esperienze e ricerche", Quaderni CIRPAS, Università degli Studi di Bari, Editore Cacucci, 2013.

8. C. Corposanto and B. Molinari, "Ma i giovani celiaci la seguono la dieta gluten free?", in C. Cipolla and A. Maturo, "Sociologia della Salute e web society", FrancoAngeli, 2013.

9. C. Corposanto and B. Molinari, "Survey e questionari online?" in C. Corposanto and A. Valastro, "Blog, fb e twitter", Giuffrè, 2014.

10. C. Cipolla, "Il ciclo metodologico della ricerca sociale", FrancoAngeli, 1997.

11. C. Corposanto, "Metodologia e tecniche non intrusive nella ricerca sociale", FrancoAngeli, 2004.

12. E.J., Webb and D.T. Campbell and R.D. Schwartw and L. Sechrest, "Unobtrusive measures - nonreactive researh in teh social sciences", Rand McNally and Co, 1966.

13. P.H. Mann, "Methods of social investigation", Blackwell, 2nd edn., 1968.

14. G. Delli Zotti, "Introduzione alla ricerca sociale. Problemi e qualche soluzione", FrancoAngeli, 2004.

15. A. Charlesworth, "Data protection, Freedom of Information and Ethical Review Committee" in Policies, Practicalities and dillemmas, Information, Communication $\&$ Society", 15, 1: 85-103. Doi: 10.1080/1369118x.2011.637572 (2012)

16. U. Volta et altri, "An Italian prospective multicenter survey on patients suspected of having non-celiac gluten sensitivity", BMC Medicine, 2014, 12:85. doi:10.1186/1741-7015-12-85 (2014).

17. H. Nissenbaum, "Privacy in Context. Technology, Policy and the Integrity of Social Life", Stanford University Press 2010.

18. Food and Drug Administration, Mobile Medical Applications, Guidance for Industry and Food and Drug Administration Staff (2013). Available: Link:

http://www.fda.gov/downloads/MedicalDevices/DeviceRegulationandGuidance/GuidanceDocuments/UCM2633 66.pdf

19. A. Trobia, "Web mining e application programming interfaces per al ricerca sociale: caratteristiche, strumenti, prospettive e limiti", in C. Corposanto and A. Valastro, "Blog, fb e twitter", Giuffrè, 2014.

20. C. Centorrino, A. Romeo, "Sociologia dei digital media", FrancoAngeli, 2012.

21. P.N., Tan and M. Steinback and V. Kumar, "Introduction to Data Mining, Reading", MA, Addison Wesley 2005.

22. A. Kaushik, "Web Analytics 2.0. Misurare il successo online nell'era del web 2.0", trad. it. Hoepli, 2012.

23. R. Brownsword, "Il consenso informato nella società dell'informazione", in C. Faralli, "Consenso informato in medicina aspetti etici e privatistici", Salute e società, XI 3: 18-41, FrancoAngeli, 2012. 